\title{
EL IMPUESTO A LA RENTA Y SU INFLUENCIA EN LA LIQUIDEZ DE LAS MICRO Y PEQUEÑAS EMPRESAS INDUSTRIALES DE CHIMBOTE, 2014
}

\section{THE INCOME TAX WING AND THEIR INFLUENCE ON THE LIQUIDITY OF THE MICRO AND SMALl INDUSTRIAL ENTERPRISES OF CHIMBOTE, 2014}

Fernando Vásquez Pacheco*

\section{RESUMEN}

T a presente investigación tuvo como objetivo general determinar y describir la Linfluencia del impuesto a la renta en la liquidez de las micro y pequeñas empresas (MYPES) industriales de Chimbote, en el año 2014. El diseño de la investigación fue no experimental, retrospectivo, correlacional y de nivel cuantitativo. La información se recabó de una muestra de 50 MYPES escogida de manera dirigida de una población de 187, a quienes se les aplicó un cuestionario de 27 preguntas cerradas utilizando la técnica de la encuesta; obteniéndose los siguientes resultados: El 78 \% de las MYPES encuestadas vienen funcionando entre 6 o más años en el sector industrial; el $92 \%$ tienen entre 3 a 15 años como empresa y el $42 \%$ se dedican a actividades relacionadas con la pesca. Por otro lado, el $70 \%$ de las MYPES encuestadas han pagado el impuesto a la renta en el año 2014 y el 30 \% no. Asimismo, para el $74 \%$, el pago del impuesto a la renta ha reducido la liquidez de sus empresas y el $76 \%$ tienen problemas con el capital de trabajo de sus empresas después que pagan el impuesto a la renta de tercera categoría. Finalmente, se ha comprobado que existe relación significativa entre el pago del impuesto a la renta por parte de las MYPES encuestadas y la liquidez de las mismas, demostrando la hipótesis planteada como verdadera.

Palabras clave: Impuesto a la renta, liquidez, MYPE.

* Facultad de Ciencias Contables financieras y Administrativas. Universidad Católica Los Ángeles de Chimbote. Teléfono 949906504. Fervaspa@yahoo.com 


\begin{abstract}
This research has the general objective: identify and describe the influence of income tax on the liquidity of micro and small enterprises (MSEs) Industrial Chimbote, in 2014. The research design was non-experimental, retrospective, correlational and quantitative level. The information was collected from a sample of 50 chosen so MYPES targeted a population of 187, who were applied a questionnaire of 27 questions closed using the survey technique; with the following results: $78 \%$ of surveyed MSEs are running between 6 or more years in the industrial sector; $92 \%$ are between 3-15 years as company and $42 \%$ are engaged in fishing related activities. On the other hand, $70 \%$ of surveyed MSEs have paid income tax in 2014 and $30 \%$ no. Also, for the $74 \%$ payment of income tax it has reduced the liquidity of their companies and $76 \%$ had problems with working capital of their companies after paying the income tax of third category. Finally, it was found that there is significant relationship between the payment of income tax by the MSEs surveyed and liquidity of the same, proving the hypothesis as true.
\end{abstract}

Keywords: Income tax, liquidity, MSEs.

\title{
INTRODUCCIÓN
}

Si se analiza la estructura productiva económica de cualquier país, sea desarrollado o en proceso de desarrollo, se constata la existencia de empresas de distinta envergadura, tales como: micro, pequeñas, medianas y grandes empresas. Asimismo, si observamos los distintos sectores económico-productivos de los diferentes países, también se da la misma dinámica; es decir, también en los distintos sectores económicos hay micro, pequeñas, medianas y grandes empresas; primando siempre, las micro y las pequeñas empresas. Al respecto, la evidencia empírica establece lo siguiente: las pequeñas empresas son una realidad indiscutible tanto en los países desarrollados como en desarrollo, porque representan la absoluta mayoría de las empresas existentes, ya que cerca del $99 \%$ del total de empresas del mundo son micro y pequeñas empresas, las mismas que generan entre el 50 y el $80 \%$ del empleo y entre el 40 y $70 \%$ del Producto Bruto Interno. Asimismo, las microempresas, representa el 95,2 \% del empresariado nacional y las pequeñas empresas el 4,8 \%. Las MYPES con ventas menores a 13 UIT son el 72,6\% $y$ en el extremo superior las MYPE con ventas mayores a 1700 UIT representan tan solo el $0.74 \%$. Asimismo, según la característica del número de trabajadores empleados: el 98,1 \% de las empresas se clasifican como microempresas y el 1,7 \% de las MYPES son pequeñas empresas. La mediana y gran empresa está representada solo por el 0,2 \%. del total de empresas (Ministerio de la Producción, 2012). La distribución geográfica de las MYPES en el Perú muestra que el 72,8 \% se ubican en las regiones de la Costa peruana, de las cuales, el 51,4 \% se localiza en Lima y Callao. Las regiones de la Sierra concentran el 21,1 \% y solo el 6,1 \% se ubican en las regiones de la Selva (Villarán, 
2007). Asimismo, tomando en cuenta la actividad económica: el 84,3 \% de las MYPES desarrollan actividades en el sector terciario de la economía, (comercio 46,9 \% y servicios $37,4 \%$ ) en el sector secundario participa el 13,1 \% (manufactura $10 \%$ y construcción $3,1 \%$ ) y, en el sector primario intervienen el 2,1 \% (1,8\% agropecuario, 0,5\% minería y el 0,3\% Pesca) (Villarán, 2007).

Por otro lado, uno de los principales problemas que tienen las MYPES en el Perú es la falta de financiamiento crediticio, lo que Flores (2006) atribuye a: 1) que las altas tasas de interés influyen en el costo de los créditos, lo que se constituye a su vez en una barrera grande para acceder al financiamiento de las actividades empresariales. 2) que faltan instituciones que avalen y apoyen nuevos proyectos, así como una cultura de riesgo en las inversiones. En general, los criterios que se aplican para otorgar financiamiento son inadecuados, pues la mayoría de los esquemas de financiamiento están diseñados para atender las necesidades de empresas grandes y medianas y no de las MYPES.

Esta situación se agrava más aún debido a lo siguiente: a) los esquemas fiscales se aplican por igual a empresas grandes, medianas, pequeñas y micro empresas, lo cual se considera un error, pues las MYPES deberían recibir un tratamiento especial. b) Los estímulos fiscales son prácticamente inexistentes. c) No se utiliza una política fiscal como agente modernizador que cambie las conductas empresariales. d) Tampoco se contempla la posibilidad de generación de empleos. e) No hay una traducción de los impuestos en buenos servicios, lo cual significa que no hay una retribución por parte del gobierno. f) La mayoría de las empresas considera que los servicios públicos son muy malos. g) La economía informal constituye una competencia desleal. h) Los empresarios perciben la existencia de un régimen de terrorismo fiscal en el que se persigue al que paga y se tolera al que no lo hace. i) Los procedimientos fiscales son largos, burocráticos y demandan una considerable inversión de tiempo y esfuerzo de los empresarios.

La situación descrita hasta aquí no es extraña en Áncash y Chimbote; lamentablemente, casi no existen estudios específicos que permitan corroborar lo que está sucediendo en el Perú, Áncash y Chimbote con relación al financiamiento de las MYPES y la política fiscal (Impuestos). No obstante, en los últimos años el estudio de las micro y pequeñas empresas ha cobrado un auge renovado durante las últimas décadas, sobre todo en países en desarrollo como el nuestro, debido a las características ya mencionadas y a la alta participación en la estructura empresarial y de empleo, son consideradas como manifestaciones vivas del potencial empresarial de las regiones (Ramírez-Urday, Mungaray y Guzmán, 2009).

Por otra parte, en toda economía de mercado y de régimen democrático se da lo siguiente: por un lado, existe una infinidad de empresas (micro, pequeñas, medianas y grandes) que necesitan (entre otros) recursos financieros (dinero) para desarrollar sus actividades productivas y de comercio de bienes y servicios. Por otro lado, los Estados (a través de los gobiernos de turno) para poder atender las necesidades de la población en sus requerimientos de bienes y servicios públicos (salud, transporte, educación, seguridad, etc.) requieren de ingresos fiscales, los cuales los obtienen a través del sistema tributario que imponen como políticas fiscales y tributarias. 
La situación descrita genera una disyuntiva entre el interés público y el interés privado; es decir, si un determinado gobierno decide incrementar los tributos de manera significativa, esto generará efectos contarios en los intereses privados, porque el Estado les estará afectando el circuito monetario de producción a los agentes empresariales, más concretamente, les estará reduciendo la disponibilidad de efectivo (liquidez) para el desarrollo de sus actividades productivas y comerciales. Al respecto, los investigadores que se ha dedicado a estudiar estos aspectos, indican lo siguiente: a) entre las causas de insolvencia de las empresas se considera: 1) La política tributaria en el país, porque el Estado en su indiscutible esfuerzo que hace para mejorar la posición de caja fiscal ha emitido normas legales antitécnicas que crean una barrera para el desarrollo empresarial; 2) las tasas de interés activas en los bancos son muy elevadas en el Perú, de modo que los costos financieros de las empresas resultan por lo general agobiantes. Estos, sumados a los sobrecostos tributarios, laborales y a la creación del impuesto del ITF, restan toda competitividad a la empresa nacional (Solórzano, 2008). b) Problemas a los contribuyentes, generándoles sobrecostos y restándoles competitividad. No solo se trata de sistemas complicados y dispersos, sino que además, afectan la liquidez de las empresas, pues los pagos adelantados no se compensan automáticamente y su devolución está sujeta a un trámite engorroso que puede durar varios meses. En ese contexto, sería muy grave que la Administración Tributaria insista en aplicar la retención al uso de tarjetas de crédito; así como en obligar a las empresas denominadas "grandes compradoras" a que cobren los embargos tributarios de sus acreedores (Alva, 2009).

En el año 2012, en la región Áncash existían 187 empresas industriales, las cuales daban empleo a 16498 trabajadores. De ellas, 58 empresas (31.02\% del total de empresas) se dedicaban a la extracción de pesca, explotación de criaderos de peces y granjas piscícolas y a otras actividades de servicios relacionadas con la pesca, 49 empresas (26.20\%) se dedicaban a la elaboración y conservación de pescado y productos de pescado (conservas de pescado). Las restantes 80 empresas (42.78 \%) se dedicaban a otras actividades industriales, tales como: elaboración y conservación de frutas, legumbres y hortalizas; elaboración de aceite y grasas de origen vegetal y animal; elaboración de productos de molinería; elaboración de productos de panadería; elaboración de azúcar; elaboración de otros productos alimenticios; elaboración de bebidas no alcohólicas, producción de aguas minerales; fabricación de otros productos minerales no metálicos; industrias básicas de hierro y acero; fabricación de productos metálicos para uso estructural; generación, captación y distribución de energía eléctrica; captación, depuración y distribución de agua y construcción de edificios completos y de partes de edificios; obras de ingeniería (Ministerio de Trabajo-Chimbote, CIIU, 2007).

El Ministerio de Trabajo, sede Chimbote, no tiene información actualizada. Según versión de un funcionario de dicha institución, ahora la información correspondiente a las empresas y a las MYPES las maneja el Ministerio de la Producción; por lo tanto, se desconoce si el pago de del impuesto a la renta por parte de las MYPES industriales de Chimbote, influye o no en la liquidez de las estas.

Por las razones expuestas y la poca información que se tiene sobre las unidades de análisis de investigación, el enunciado del problema de investigación es el siguiente: 
¿Cómo influye el impuesto a la renta en la liquidez de las micro y pequeñas empresas industriales de Chimbote en el año 2014? Para dar respuesta a este enunciado, se ha establecido el siguiente objetivo general: Determinar y describir la influencia del impuesto a la renta en la liquidez de las micro y pequeñas empresas industriales de Chimbote, año 2014. Para poder conseguir este objetivo, planteamos los siguientes objetivos específicos: 1. Describir las principales características de las micro y pequeñas empresas industriales de Chimbote, año 2014. 2. Describir las principales características de los tributos que pagan las micro y pequeñas industriales de Chimbote, año 2014. 3. Describir las principales características de la liquidez financiera de las micro y pequeñas empresas industriales de Chimbote, año 2014. 4. Determinar si el pago del impuesto a la renta de tercera categoría por parte de las micro y pequeñas industriales de Chimbote afecta su liquidez, año 2014.

Asimismo, planteamos la siguiente hipótesis: El pago del impuesto a la renta por parte de las micro y pequeñas empresas industriales de Chimbote influye significativamente en la liquidez financiera de las mismas, año 2014.

Finalmente, la presente investigación se justifica por las siguientes razones: En el Perú, la gran mayoría de empresas son micro y pequeñas empresas, así por ejemplo las MYPES según el número de trabajadores son el $99.8 \%$; en consecuencia, solo el $0.2 \%$ son medianas y grandes empresas. Desagregando esta información se tiene: $98.1 \%$ son micro empresas, de las cuales el $96.3 \%$ tiene de la 5 trabajadores y el $1.8 \%$ tiene entre 6 a 10 trabajadores. Asimismo, $1.7 \%$ son pequeñas empresas, de las cuales el $1 \%$ tiene de 11 a 20 trabajadores, el $0.5 \%$ de 21 a 50 trabajadores y el $0.2 \%$ de 51 a 100 trabajadores. 2) Las MYPES generan empleo para el $62 \%$ de la fuerza laboral del país, en tanto que la mediana y gran empresa contribuye con el $7 \%$ de los empleos, el $19 \%$ corresponde a los independientes, el $8 \%$ al sector público y el $4 \%$ a trabajadores del hogar. 3) Por actividad económica, el $84.2 \%$ de las MYPES desarrollan actividades en el sector terciario de la economía (47.2 \% en comercio y $37 \%$ en servicios); en el sector secundario participan el 13.1 \% (manufactura $10.3 \%$ y $2.8 \%$ en el sector construcción); y en el sector primario, el $2.7 \%$ ( $1.9 \%$ en el sector agropecuario, $0.5 \%$ en minería y $0.3 \%$ en pesca). 4) El Perú es considerado como uno de los países con mayor generación de iniciativas emprendedoras en el mundo; también es el primer país en mortalidad de empresas, se estima que el 50 \% de la MYPES quiebran antes de llegar al primer año, situación que se origina por una falta de conocimiento de las herramientas de gestión y habilidades gerenciales, y que se agrava por la elevada informalidad de sus operaciones, improvisación, baja productividad y, en consecuencia baja competitividad, que impacta en una menor rentabilidad, y termina con la quiebra. Los factores que más inciden en esta problemática son la escasa capacidad operativa, la limitada capacidad gerencial, los problemas para contar con información adecuada y oportuna, la desarticulación empresarial, la inadecuada tecnología y la dificultad de acceso al crédito (Ravelo, s/f).

Las razones precedentes denotan que las MYPES en el Perú son importantes desde el punto de vista cuantitativo y cualitativo. Además, estas empresas también se enfrentan al sistema tributario imperante en el País, el mismo que seguramente en su aplicación y operatividad le genera mayores efectos que a las medianas y grandes empresas, por 
el hecho de que las MYPES tienen serios problemas de financiamiento, capacitación, competitividad y rentabilidad; por lo tanto, el pago de impuestos les generarán mayores efectos negativos que a las medianas y grandes empresas; en consecuencia, es importante estudiar la problemática que genera el pago de tributos (impuestos) en los aspectos financieros (liquidez) de las micro y pequeñas empresas industriales de Chimbote.

\section{MATERIAL Y MÉTODOS}

El diseño de la investigación fue no experimental, retrospectivo, correlacional y de nivel cuantitativo. La información se recabó de una muestra de 50 micro y pequeñas empresas industriales, escogida de manera dirigida de una población de 187, a quienes se les aplicó un cuestionario de 27 preguntas cerradas utilizando la técnica de la encuesta.

\section{RESULTADOS}

\section{Respecto al objetivo específico 1:}

Tabla 1. Principales características de las MYPES industriales de Chimbote.

\begin{tabular}{|c|c|}
\hline Ítems & Resultados \\
\hline $\begin{array}{l}\text { ¿Desde cuándo su empresa está en el } \\
\text { sector industrial? }\end{array}$ & $\begin{array}{l}\text { El } 78 \text { \% (39) de las empresas encuestadas } \\
\text { tiene en el sector industrial una antigüedad } \\
\text { entre } 6 \text { a más años en el sector y el rubro } \\
\text { y el } 22 \% \text { (11) tienen entre } 3 \text { a } 5 \text { años. }\end{array}$ \\
\hline $\begin{array}{l}\text { ¿Cuántos trabajadores laboran en su } \\
\text { empresa? }\end{array}$ & $\begin{array}{l}\text { El } 60 \% \text { (30) de las empresas encuestadas } \\
\text { tiene entre } 1 \text { a } 10 \text { trabajadores y el } 40 \% \\
\text { (20) entre } 11 \text { y más. }\end{array}$ \\
\hline ¿A qué se dedica su empresa? & $\begin{array}{l}\text { El } 42 \%(21) \text { de las empresas encuestadas } \\
\text { se dedica a desarrollar actividades } \\
\text { relacionadas con la pesca, el resto } 58 \% \\
\text { (29) se dedica a actividades de: cultivos de } \\
\text { productos agrícolas, fabricación de prendas } \\
\text { de vestir, entre otras }\end{array}$ \\
\hline ¿Desde cuándo su empresa es formal? & $\begin{array}{l}\text { El } 92 \% \text { (46) de las empresas encuestadas } \\
\text { tiene entre } 3 \text { a } 15 \text { años como empresas } \\
\text { formales y el } 8 \% \text { (4) tiene entre } 1 \text { a } 2 \text { años. }\end{array}$ \\
\hline $\begin{array}{l}\text { ¿Su empresa ha tenido utilidades en } \\
\text { los dos últimos años: } 2012 \text { ? }\end{array}$ & $\begin{array}{l}\text { El } 98 \%(49) \text { de las empresas encuestadas } \\
\text { ha tenido utilidades en los } 2 \text { últimos años } \\
(2013,2014) .\end{array}$ \\
\hline
\end{tabular}

Fuente: Elaboración propia en base a la aplicación del cuestionario. 


\section{Respecto al objetivo específico 2}

Tabla 2. Principales características de los tributos que pagan las micro y pequeñas empresas industriales de Chimbote.

Ítems

¿Está de acuerdo con el pago del Impuesto a la Renta (IR)?

\section{Resultados}

El $76 \%$ (38) de las empresas encuestadas no está de acuerdo con el pago del impuesto a la renta y el $24 \%$ (12) si está de acuerdo con dicho pago.

¿En el año 2014 ha tenido ingresos netos en la empresa? El $70 \%$ (35) de las empresas encuestadas ha tenido en el año 2013 ingresos netos en el desarrollo de sus actividades productivas y el $30 \%$ (15) no ha tenido ingresos netos.

¿En el año 2014 ha pagado el El $70 \%$ (35) de las empresas encuestadas Impuesto a la Renta? ha pagado el impuesto a la renta en el año 2013 y el 30 \% (15) no ha pagado dicho impuesto.

¿Qué porcentaje pago de Impuesto a la Renta en el año 2014?

El $70 \%$ (35) de las empresas encuestadas ha pagado e una tasa del $1.5 \%$ del impuesto a la renta y el 30 \% (15) no precisa.

¿Qué efectos le genera en su empresa el pago del Impuesto a la Renta de 3ra Categoría?

El $74 \%$ (37) de los empresarios encuestados dijo que el pago del impuesto a la renta reduce la liquidez de la empresa y el $26 \%$ (13) dijo que reduce el capital de trabajo.

¿Cómo paga el IR de 3ra. Categoría? Adelantado?

El $80 \%$ (40) de las empresas encuestadas paga el impuesto a la renta adelantado y el 20 \% (20) paga a fin de año (anual).

¿Está de acuerdo con el porcentaje $\quad$ El $64 \%$ (32) de las empresas encuestadas (\%) del IR que paga? no está de acuerdo con el porcentaje del impuesto a la renta que paga y el $36 \%$ (18) sí está de acuerdo.

¿El pago del IR de 3ra. Categoría le genera problemas en la liquidez de su empresa?

El 96 \% (48) de los empresarios encuestados dijo que el pago del impuesto a la renta de tercera categoría sí les genera problemas en la liquidez de sus empresas y solo el 4 \% (2) dijo que no.

Fuente: Elaboración propia en base a la aplicación del cuestionario. 


\section{Respecto al objetivo específico 3}

Tabla 3. Principales características de los tributos que pagan las micro y pequeñas empresas industriales de Chimbote.

\begin{tabular}{|c|c|}
\hline Ítems & Resultados \\
\hline $\begin{array}{l}\text { ¿Su empresa solicita financiamiento } \\
\text { crediticio de terceros? }\end{array}$ & $\begin{array}{l}\text { El } 100 \% \text { (50) de los empresarios encuestados } \\
\text { dijo que sí solicita créditos financieros a } \\
\text { terceros. }\end{array}$ \\
\hline $\begin{array}{l}\text { Durante el proceso productivo y de } \\
\text { comercialización, su empresa tiene } \\
\text { problemas de liquidez }\end{array}$ & $\begin{array}{l}\text { El } 80 \% \text { (40) de los empresarios encuestados } \\
\text { dijo que sí tiene problemas de liquidez en el } \\
\text { proceso productivo y de comercialización y el } \\
20 \% \text { (10) dijo que no. }\end{array}$ \\
\hline $\begin{array}{l}\text { ¿Después que paga el IR, tiene } \\
\text { problemas con el capital de trabajo } \\
\text { en su empresa? }\end{array}$ & $\begin{array}{l}\text { El } 76 \% \text { (38) de los empresarios encuestados } \\
\text { dijo que después del pago del impuesto a la } \\
\text { renta tiene problemas con el capital de trabajo } \\
\text { en sus empresas y el } 24 \% \text { (12) dijo que no. }\end{array}$ \\
\hline $\begin{array}{l}\text { ¿Después que paga el IR, tiene } \\
\text { problemas para pagar a sus } \\
\text { acreedores? }\end{array}$ & $\begin{array}{l}\text { El } 50 \% \text { (25) de los empresarios encuestados } \\
\text { dijo que después de pagar el impuesto a } \\
\text { la renta tiene problemas para pagar a sus } \\
\text { acreedores y el } 50 \% \text { (25) dijo que no. }\end{array}$ \\
\hline $\begin{array}{l}\text { ¿En el } 2014 \text { el pago del IR, le genera } \\
\text { disminución en la liquidez de su } \\
\text { empresa? }\end{array}$ & $\begin{array}{l}\text { El } 80 \% \text { (40) de los empresarios encuestados } \\
\text { dijo que el pago del impuesto a la renta le } \\
\text { genera disminución en la liquidez de sus } \\
\text { empresas en el año } 2014 \text { y el } 20 \% \text { (10) dijo } \\
\text { que no. }\end{array}$ \\
\hline $\begin{array}{l}\text { ¿La disminución de la liquidez } \\
\text { por el pago del IR es significativa } \\
\text { (importante)? }\end{array}$ & $\begin{array}{l}\text { El } 94 \text { \% (47) de los empresarios encuestados } \\
\text { dijo que la disminución de la liquidez en } \\
\text { sus empresas es significativo por el pago del } \\
\text { impuesto a la renta y el } 6 \% \text { (3) dijo que no. }\end{array}$ \\
\hline $\begin{array}{l}\text { ¿Cómo soluciona el problema de } \\
\text { la disminución de la liquidez de su } \\
\text { empresa por el pago de IR? }\end{array}$ & $\begin{array}{l}\text { El } 56 \text { \% (28) de los empresarios encuestados } \\
\text { dijo que una solución al problema de la } \\
\text { disminución de la liquidez por el pago } \\
\text { del impuesto a la renta es recurriendo al } \\
\text { financiamiento de terceros y el } 44 \text { \% (22) dijo } \\
\text { que lo hacen a través de autofinanciamiento. }\end{array}$ \\
\hline
\end{tabular}

Fuente: Elaboración propia en base a la aplicación del cuestionario. 


\section{Respecto al objetivo específico 4}

Se encontró una Chi Cuadrado de 12.578, siendo -con un grado de libertad igual a uno- mayor de 3.84, significando que $X_{0}^{2}>X_{t}^{2}$, entonces, se rechaza $\mathrm{H}_{0}$ y se acepta $\mathrm{H}_{\mathrm{a}}$; por lo tanto, existe relación entre las variables pago del impuesto a la renta y la liquidez. $(p=0.05)$

\section{DISCUSIÓN}

\section{Respecto a los resultados del objetivo específico 1:}

1. El $78 \%$ de las MYPES encuestadas tienen una antigüedad entre 6 y más años en el sector industrial y el $22 \%$ tienen entre 3 a 5 años de antigüedad. Estos resultados indican que las MYPES encuestadas tienen la antigüedad suficiente para ser estables (Avolio, Mesones y Roca, s/f).

2. El $92 \%$ de las empresas encuestadas tiene entre 3 a 15 años como empresas formales y el $8 \%$ tiene entre 1 a 2 años. Estos resultados indican que casi el $100 \%$ de la empresas encuestadas tienen la antigüedad suficiente como empresas formales, lo que seguramente les genera ventajas en el acceso al financiamiento, a participar para obtener la buena pro en concursos para abastecer productos industriales al Estado, vender y comprar tanto en los mercados internos como en los mercados internacionales. Sin embargo, la formalización empresarial no necesariamente garantiza que las empresas industriales sean competitivas en los mercados internacionales, porque las exportaciones dependen fundamentalmente de: costos, precios y calidad de los productos que se exportan. Por el contrario, es posible que por los impuestos pagados (entre otros) estas empresas sean menos competitivas en dichos mercados, lo que a su vez podría estar ocasionando falta de recursos para hacer estudios de mercados, investigación, etc. (Sociedad Peruana de PYME y Román, 2013).

3. El $60 \%$ de las empresas encuestadas tiene entre 1 a 10 trabajadores y el $40 \%$ tienen entre 11 y más trabajadores permanentes. Estos resultados indican que desde el punto de vista del número de trabajadores, el $60 \%$ de las MYPE encuestadas son microempresas y el $40 \%$ son pequeñas empresas. (Toledo, 2009)

4. El $42 \%$ de las empresas encuestadas se dedican a actividades relacionadas con la pesca, el resto (58 \%) a otras actividades industriales, tales como: cultivos de productos agrícolas, fabricación de prendas de vestir, panadería, entre otras.

5. En promedio, el $98 \%$ de las empresas encuestadas ha tenido utilidades en los 2 últimos años $(2013,2014)$. Estos resultados indican que casi el $100 \%$ de las empresas encuestadas en los años 2013 y 2014 han tenido utilidades; por lo tanto, tienen la obligación de pagar el impuesto a la renta., conforme lo establece el Decreto Legislativo N. ${ }^{\circ} 1120$ de fecha 18-07-12 y que entró en vigencia el 01-01-13. Este Decreto modificó el Art. $85^{\circ}$ de la LIR, Tercera Disposición Complementaria Final y Cuarta Disposición Complementaria Transitoria-Pagos a Cuenta del Impuesto a la Renta de Tercera Categoría, en los siguientes términos: respecto al denominado sistema de porcentajes, se 
ha disminuido el porcentaje del cálculo que se venía haciendo, pasando este del $2 \%$ al $1.5 \%$ mensual; por lo tanto, dicha medida fue favorable al contribuyente, toda vez que contribuye al cumplimiento de las obligaciones tributarias, sobre todo si se trata de incorporar a los sectores donde los índices de evasión es alto. (Caballero Bustamante, s/f)

\section{Respecto a los resultados del objetivo específico 2:}

1. El $76 \%$ de las empresas encuestadas no está de acuerdo con el pago del impuesto a la renta y el $24 \%$ si están de acuerdo con dicho pago. Estos resultados indican que más de dos tercios de las empresas encuestadas no están de acuerdo con el pago del impuesto a la renta. Es posible que ello se deba a que el pago de dicho impuesto genera problemas de liquidez en sus empresas, más aún, por el hecho de que el pago del impuesto a la renta es de carácter adelantado (La República, 2012).

2. El $70 \%$ de las empresas encuestadas ha tenido en el año 2014 ingresos netos en el desarrollo de sus actividades productivas y el $30 \%$ no han tenido ingresos netos. Estos resultados indican que más de 2/3 (70 \%) de los empresarios encuestados ha tenido ingresos netos en el año 2014; por lo tanto, ha tenido utilidades y consecuentemente, tienen que haber pagado el impuesto a la renta.

3. El $70 \%$ de las empresas encuestadas ha pagado el impuesto a la renta en el año 2014 y el 30 \% no han pagado dicho impuesto. Las implicancias del hecho de que el 70 \% de las empresas en el año 2014 han pagado sus impuestos serían: por un lado, no tendrán problemas de multas y amonestaciones por parte de Sunat (s/f); en cambio, el 30 \% de empresarios que no ha pagado el impuesto a la renta sí tendrán problemas como los descritos.

4. El $70 \%$ de las empresas encuestadas ha pagado una tasa de $1.5 \%$ del impuesto a la renta y el $30 \%$ no precisan. Estos resultados estarían indicando también los efectos del Decreto Legislativo $N^{\circ}$ 1120. (Caballero Bustamante, 2013)

5. El $74 \%$ de los empresarios encuestados dijo que el pago del impuesto a la renta reduce la liquidez de la empresa y el $26 \%$ dijo que reduce el capital de trabajo. Estos resultados estarían indicando que para el $100 \%$ de los empresarios encuestados, el pago del impuesto a la renta reduce la liquidez de sus empresas, porque la reducción del capital de trabajo es otra forma de decir que se reduce la liquidez (Albornoz, 2006).

6. El $80 \%$ de las empresas encuestadas paga el impuesto a la renta adelantado y el $20 \%$ pagan a fin de año (anual). Sin embargo, las implicancias de estos resultados serían diferentes: para el $80 \%$ de empresarios que ha pagado el impuesto a la renta de manera adelantada, los efectos en la liquidez serán mayores; en cambio, el 20 \% de empresarios que ha pagado el impuesto a la renta a fin de año, los efectos en la liquidez empresarial será menor que en el primer caso (Caballero Bustamante, 2013).

7. El $64 \%$ de las empresas encuestadas no está de acuerdo con el porcentaje del impuesto a la renta que pagan y el 36 \% sí está de acuerdo. Las implicancias de estos resultados podrían ser contradictorios; por un lado, para el $64 \%$ de empresarios que 
han manifestado de que no están de acuerdo con el porcentaje de impuesto que pagan, es porque seguramente lo han evidenciado en la práctica, porque están acostumbrados a pagar el impuesto a la renta; en cambio, el $36 \%$ que dice que no, posiblemente no pagan impuesto, y si lo hacen, es esporádicamente.

8. El $96 \%$ de los empresarios encuestados dijo que el pago del impuesto a la renta de tercera categoría sí les genera problemas en la liquidez de sus empresas y solo el 4 \% dijo que no. Estos resultados estarían confirmando lo que la teoría establece y la evidencia empírica demuestra. (Berneo, 2014; Álvarez y Dionicio 2008; La República, 2012 y Conexión Esan, 2012).

\section{Respecto a los resultados del objetivo específico 3:}

1. Todos los empresarios encuestados dijeron que sí solicitan créditos financieros a terceros. Estos resultados estarían implicando que los empresarios por el hecho de ser formales y tener la mayoría más de 2 años como formales, no tienen problemas para acceder al sistema financiero para obtener financiamiento crediticio; sin embargo, ello no necesariamente garantiza que los empresarios hayan obtenido los créditos financieros en los montos solicitados y a las tasas de interés adecuadas. (Superintendencia de Banca y seguro, s/f.)

2. El $80 \%$ de los empresarios encuestados dijo que sí tienen problemas de liquidez en el proceso productivo y de comercialización, y el 20 \% dijo que no. Las implicancias de estos resultados podrían ser explicados con los mismos argumentos establecidos en el ítem anterior.

3. El $76 \%$ de los empresarios encuestados dijo que después del pago del impuesto a la renta sí tienen problemas con el capital de trabajo en sus empresas y el $24 \%$ dijo que no. Estos resultados estarían indicando que el pago del impuesto a la renta: al $76 \%$ de los empresarios les ocasiona problemas con el capital de trabajo de que disponen para el desarrollo cotidiano de sus actividades productivas y de comercialización, y al 24 \% no les genera este tipo de problemas. Estos resultados son más o menos congruentes con los resultados del ítem anterior, porque en realidad, la liquidez y el capital de trabajo, en el fondo son lo mismo, la diferencia es solo de tecnicismo.

4. El $50 \%$ de los empresarios encuestados dijo que después de pagar el impuesto a la renta tienen problemas para pagar a sus acreedores y el $50 \%$ dijeron que no. Estos resultados estarían implicando que la mitad de los empresarios encuestados si tienen problemas con sus acreedores por el hecho de pagar el impuesto a la renta y la otra mitad no tienen problemas. Las posibles explicaciones podrían ser: para las empresas que tienen problemas, podría ser que la mayoría son micro y, por lo tanto, tienen dificultades para acceder a los créditos en los montos solicitados; es posible que el rubro del negocio sea tal que los flujos de retorno de dinero no sean continuos y permanentes; es posible que los empresarios en cuestión tengan muchos acreedores, etc. Para los empresarios que no tienen problemas, la explicación sería sentido contrario. 
5. El $74 \%$ de los empresarios encuestados dijo que el pago del impuesto a la renta les genera disminución en la liquidez de sus empresas y el 26 \% dijo que no. En el caso del $74 \%$ de los empresarios que dijeron que el pago del impuesto a la renta disminuye la liquidez de sus empresas, es congruente con lo que establece la teoría y algunos trabajos empíricos revisados. (Berneo, 2014; Álvarez y Dionicio 2008; La República, 2012 y Conexión Esan, 2012). Aquí el problema es con el 26 \% de empresarios que dijeron que el pago del impuesto a la renta no disminuye la liquidez de sus empresas, En este caso, se podría especular diciendo que es posible que ello se deba a lo siguiente: son empresas que tienen la suficiente liquidez en todos los procesos productivos involucrados, contestaron la pregunta mecánicamente y dijeron que no, el rubro del negocio es tal que permanentemente tienen flujos de ingreso de dinero, etc.

6. El $80 \%$ de los empresarios dijo que el pago del impuesto a la renta les generó una disminución en la liquidez de sus empresas en el año 2014 y el 20 \% dijo que no. Estos resultados confirman lo que dicen varios autores e instituciones (Berneo, 2014; Álvarez y Dionicio 2008; La República, 2012 y Conexión Esan, 2012).

7. El $94 \%$ de los empresarios encuestados dijo que la disminución de la liquidez en sus empresas es significativo por el pago del impuesto a la renta y el $6 \%$ dijo que no. Estos resultados estarían indicando que por el hecho de ser micro y pequeñas empresas, generalmente tienen problemas para obtener financiamiento apropiado (Conexión Esan, 2012).

8. El $56 \%$ de los empresarios encuestados dijo que una solución al problema de la disminución de la liquidez por el pago del impuesto a la renta es recurriendo al financiamiento de terceros y el $44 \%$ dijo que lo hace a través de autofinanciamiento. Esta forma de comportamiento de los empresarios frente a la misma situación problemática (disminución de liquidez por pago del impuesto a la renta), podría estar siendo explicada por el rubro del negocio, por la disponibilidad de efectivo propio, o por los altos costos que implica el financiamiento del sistema financiero (Conexión Esan, 2012)

\section{Respecto a los resultados del objetivo específico 4:}

Los resultados de la chi cuadrado indican que existe relación significativa entre el pago del impuesto a la renta y la liquidez de las MYPE industriales de Chimbote. Es decir, para los 50 empresarios encuestados se encontró asociación (relación) estadísticamente significativa entre el pago del impuesto a la renta y la liquidez de sus empresas, con una probabilidad de error menor o igual al $5 \%(\mathrm{p} \leq 0.05)$.

\section{CONCLUSIONES}

\section{Respecto al objetivo específico 1:}

Todas las MYPE industriales encuestadas de Chimbote tienen una antigüedad en el sector de más de 3 años. Asimismo, poco más de la mitad (60 \%) son micro empresas y el resto (40 \%) son pequeñas empresas; cerca de la mitad (42\%) se dedican a la industria 
relacionada con la pesca y poco más de la mitad (58 \%), se dedican a otras actividades industriales, tales como: cultivos y transformación de productos agrícolas, fabricación de prendas de vestir, panadería, entre otras. Finalmente, el total de las empresas encuestadas (100 \%) son formales y tienen más de 2 años como formales. Asimismo, casi la totalidad de las MYPES encuetadas (98 \%), ha tenido utilidades en los años 2012 y 2013.

\section{Respecto al objetivo específico 2:}

Poco más de 2/3 de las MYPES encuestadas (74\%), conoce las leyes tributarias y el resto (26\%), no las conoce. También, poco más de 2/3 de las MYPES encuestadas (74 \%), conoce la ley del impuesto a la renta y el resto (26\%), no la conoce. Asimismo, poco más de 2/3 de las MYPES encuestadas (74 \%), no está de acuerdo con el pago del impuesto a la renta y el resto (26\%), sí está de acuerdo. En el año 2014 poco más de 2/3 de las MYPE encuestadas (70 \%) ha tenido ingresos netos en el desarrollo de sus actividades productivas y el resto (30 \%), no ha tenido ingresos netos. También, poco menos de 2/3 (60 \%) ha pagado $1.5 \%$ mensual como tasa del impuesto a la renta; la mayoría (80 \%) pagan el impuesto a la renta adelantado y el resto $(20 \%)$ paga a fin de año. Finalmente, para poco más de $2 / 3$ de las MYPES encuestadas (74 \%), el pago del impuesto a la renta reduce la liquidez de sus empresas y para el resto (26\%), reduce el capital de trabajo; aproximadamente 2/3 (64\%) está de acuerdo con la tasa que paga del impuesto a la renta y el resto (36 \%) no está de acuerdo, y para casi la totalidad (96 \%) el pago de la renta de tercera categoría le genera problemas en la liquidez de sus empresas.

\section{Respecto al objetivo específico 3:}

La totalidad de las MYPES encuestadas (100\%), solicita créditos financieros a terceros; Asimismo, a mayoría (80 \%) tienen problemas de liquidez en el desarrollo de sus actividades productivas y de comercialización, el resto $(20 \%)$ no tienen dichos problemas. También, poco más de 2/3 (76 \%) tiene problemas con el capital de trabajo de sus empresas y el resto (24\%), no tiene problemas; para poco más de 2/3 encuestadas (74 \%) el pago del impuesto a la renta disminuye la liquidez de sus empresas y para el resto (26\%) dicho pago no disminuye la liquidez de sus empresas. Finalmente, en el año 2014, para la mayoría de las MYPES encuestadas (80 \%) el pago del impuesto a la renta disminuye la liquidez de sus empresas y para el resto (20\%) no. Asimismo, casi para la totalidad (94 \%), la disminución en la liquidez de sus empresas fue significativa por el pago del impuesto a la renta.

Respecto al objetivo específico 4: Existe relación significativa entre el pago del impuesto renta y la liquidez financiera de las MYPE encuestadas. 


\section{REFERENCIAS BIBLIOGRÁFICAS}

Alva, E. (2009). El Régimen de los pagos adelantados del Impuesto General a las Ventas por concepto de percepciones a las importaciones y su efecto en la liquidez en las empresas importadoras del sector plástico en el departamento de Lima durante los años 2007-2008. Tesis para optar el grado de Maestría en Contabilidad. Universidad Nacional Mayor de San Marcos. Escuela de Posgrado. Unidad de posgrado de la Facultad de Ciencias Contables, Lima.

Álvarez, M., Dionicio, G. (2008). Influencia de la Aplicación del Sistema de Pago de Obligaciones Tributarias con el Gobierno Central en la Situación Económica y Financiera de la Empresa Transialdir SAC en el bienio 2006-2007. Tesis para optar el título profesional de Contador Público. Facultad de Estudio de la Empresa, Carrera de Contabilidad f Finanzas, Universidad Privada del Norte, Trujillo.

Avolio, B., Mesones, A. y Roca, E. (s/f). Factores que limitan el crecimiento de las micro y pequeñas empresas en el Perú (MYPES). Tomado (20-04-14) de: http://scholar. google.es/scholar?hl=es\&q= \%28Avolio \%2C+B. \%2C+Mesones \%2C+A.+y++Roc a $\% 2 C+E .+\& b t n G=\& l r=$

Alvornoz, C. (2006). Capital de trabajo un tema importante para considerar en las empresas. En Publicaciones Universo-Económico. Tomado (23-04-14) en: http:// www.consejo.org.ar/publicaciones/ue/ue78/trabajo.htm

Avolio, B., Mesones, A. y Roca, E. (s/f). Factores que limitan el crecimiento de las Micro y Pequeñas Empresas en el Perú (MYPE). CENTRUM Católica. Lima.

Bermeo, E. (2014). Influencia del anticipo del impuesto a la renta en el flujo de caja aplicado al sector comercial, caso: Sanilsa Distribuidora de Alimentos Cia. Ltda., año 2012. Tesis para obtener el título de Ingeniero en Contabilidad y Auditoría. Universidad Politécnica Salesiana- Sede Cuenca.

Caballero BustamenteE (s/f). Informativo Caballero Bustamante, icb. Tomado (23-0414) de: www.cabalerobustamante.com.pe pantilla/2012/DL1120_Rentapdf

Conexión ESAN (2012). Polémica por las tasas de interés a las Mypes. Tomado (24-0414) de: http://www.esan.edu.pe/conexion/actualidad/2012/11/02/tasas-de-interesmypes/

Flores, A. (2006). Metodología de la gestión para la micro, pequeñas y medianas empresas en Lima Metropolitana. Tesis doctoral, Universidad Nacional Mayor de San Marcos, Lima.

La República (2012). Pymes pagarán más de 50 \% de Impuesto a la renta. Tomado (2404-14) de: http://www.larepublica.pe/05-09-2012/pymes-pagaran-hasta-50-masen-impuesto-la- renta

Ministerio de Trabajo - Chimbote CIIU (2007). Catálogo de empresas industriales. 
Ravelo, J. (2012). Las mypes en el Perú. Strategia, (26), 45-

47 (Tomado: 25-02-14) de:http://scholar.google.es/ scholar?hl=es\&q=Ravello \%2C+Mypes+en+el+Peru\&btnG=\&lr=

Ramírez-Urday, M., Mungaray, A. y Guzman N. (2009). Restricciones de liquidez en microempresas y la importancia del financiamiento informal en Baja California. Revista Región y Sociedad. V. 21. № 44, México ene/abr.

Román, M. (2013). ¿Por qué fracasan las empresas? En Perú 21. PE. Tomado (24-04-14) de: http://peru21.pe/mis-finanzas/que-fracasan-empresas-2131876

Sociedad Peruana de PYME. (2013). ¿Por qué fracasan las empresas? En Perú21. PE. Tomado (24-04-14) de: http://peru21.pe/mis-finanzas/que-fracasan-empresas-2131876

Superintendencia de Banca y Seguros (s/f). Gestión. Tomado el (23-04-14) de: http:// gestion.pe/noticia/629801/mypes-pagan-31-credito-gran-empresa-solo9

Sunat (s/f). (Extraido 09 mar 2014) de: http://www.sunat.gob.pe/legislacion/renta/regla/

Toledo, E. (2009). Microfinanzas: diagnóstico del sector de la micro y pequeña empresa y su tecnología crediticia. Banco Financiero del Perú. División de micro-finanzas y pequeña empresa. Contabilidad y Negocios.

Villarán, F. (2007). El mundo de la pequeña empresa. Copeme-Confiep-Mincetur. 